\title{
A MICS Band Wireless Body Sensor Network
}

\author{
Mehmet R. Yuce, Steven W. P. Ng ${ }^{1}$, Naung L. Myo ${ }^{1}$, Chin K. Lee ${ }^{1}$, Jamil Y. Khan ${ }^{1}$, and Wentai Liu ${ }^{2}$ \\ ${ }^{I}$ The School of Electrical Eng. and Computer Sci., The University of Newcastle, Callaghan, NSW 2308, Australia. \\ ${ }^{2}$ Electrical and Computer Eng., University of California, Santa Cruz, CA 95064-1077, USA.
}

\begin{abstract}
This paper presents a MICS (Medical Implant Communication Service) based body sensor network design and implementation for patient physiological data collection for health monitoring purposes. The MICS band offers the advantage of miniaturized electronic devices that can either be used as an implanted node or as an external node. In this work, a prototype sensor network is implemented by incorporating temperature and pulse rate sensors on nodes. Each developed sensor node has the capability of physiological data acquisition and local processing. The sensor node can also transmit data over the air to a remote central control unit $(\mathrm{CCU})$ for further processing and storage. The developed system offers medical staff to obtain patient's physiological data on demand basis via the Internet. Moreover, the system has been optimized for power consumption by using a polling architecture. Some preliminary performance data is presented in the paper.
\end{abstract}

Index Terms - MICS band, medical network, telemedicine.

\section{INTRODUCTION}

According to a NEW YORK TIMES report, 5\% of people admitted to hospitals, or about 1.8 million people per year, in the United States pick up an infection while staying there. Such infections are induced by physicians, hospitals, drugs, and medical procedures. They are directly responsible for the 20,000 deaths among hospital patients in the U.S. each year, and they have effect on an additional 70,000 deaths, according to the federal Centers for Disease Control (CDC) [1]. The dollar cost of such infections estimated by the CDC is $\$ 4.5$ billion. In most hospitals, the number of medical staff is not sufficient. Doctors and nurses often work longer than they are expected and even work double shifts, making them more prone to errors. With increasing number of aging population in most part of the world, healthcare system becomes a global issue.

With the advancement of wireless technologies, high performance and fault tolerant wireless devices can be employed to eliminate medical errors, to reduce workload, to improve the efficiency of hospital staff as well as to improve the comfort of patients. Thus, over the past decade, there has been increased interest among researchers in developing wireless recording and monitoring for real-time physiological parameters (e.g. ECG, EEG, EOG, EMG, Neural, Blood Flow, Blood Pressure etc.) from a patient body in medical environments [2]-[7]. it is now possible to use the advanced wireless technology to introduce safe and reliable wireless systems in healthcare area. With successful implementation of such projects, patients in hospital can now sleep in peace without having nurses to wake them up to take samples of their physiological body parameters. Doctors and nurses will no longer have to go to the ward and do routine checks and yet have the patient's condition monitored.

Previous wireless data collection systems [2]-[7] used standards such as Zigbee or Bluetooth that do not comply with the medical standard due to their size, power consumption and strong interference from other devices. Considering hundred sensors attached to a patient's body, such systems become quite bulky to be carried by patients. Therefore, the USA (FCC) and other countries communication authorities have recently allocated a new band Medical Implant Communication Service band (MICS) at 402-405 MHz with $300 \mathrm{KHz}$ channels to enable the wireless communication of such medical devices to deliver high level of comfort, mobility and better patient care [8][9]. In addition, the 402-405 MHz frequencies have propagation characteristics conducive to the transmission of radio signals within human body and do not pose as significant risk of interference to other radio operations in that band [10]. The MICS is an ultra-low power, unlicensed, mobile radio service for transmitting data to support diagnostic or therapeutic functions associated with implanted medical devices. The MICS band permits individuals and medical practitioners to utilize ultra-low power medical implant devices, such as cardiac pacemakers and defibrillators, without causing interference to other users of the electromagnetic radio spectrum [8][9][13].

The aim of this project is to build a Wireless Body Sensor Network (WBSN) that is based on the newly available 402$405 \mathrm{MHz}$ MICS band. As mentioned above, this band was particularly chosen to provide small size, low-power, faster data transfer and longer communication range. The MICS band is located at an optimum frequency range that promises high-level of integration with the advanced radio frequency IC (RFIC) technology. This results in miniaturization and lowpower consumption. While higher frequency causes higher penetration loss, high-level integration becomes difficult at low frequencies. In addition, there exist relatively insignificant penetration loss at these frequencies $(10 \mathrm{~dB}$ with $10 \mathrm{~mm}$ tissue penetration)[10]. Moreover, a small antenna design is also difficult at lower frequencies. The antennas for this project are designed as a loop printed around the prototyping boards (See Fig. 7). Combining all these features with the availability of the 402-405 MHZ band internationally offers an attractive frequency choice for the targeted WBSN application.

In the following section, an overview of the Wireless Body Sensor Network is presented; followed by the description of sensor nodes. In Section IV, the CCU is discussed in details. Section VI discusses the firmware and software design and methods for data packing to achieve low power consumption. Conclusions and future work is discussed in Section VI. 


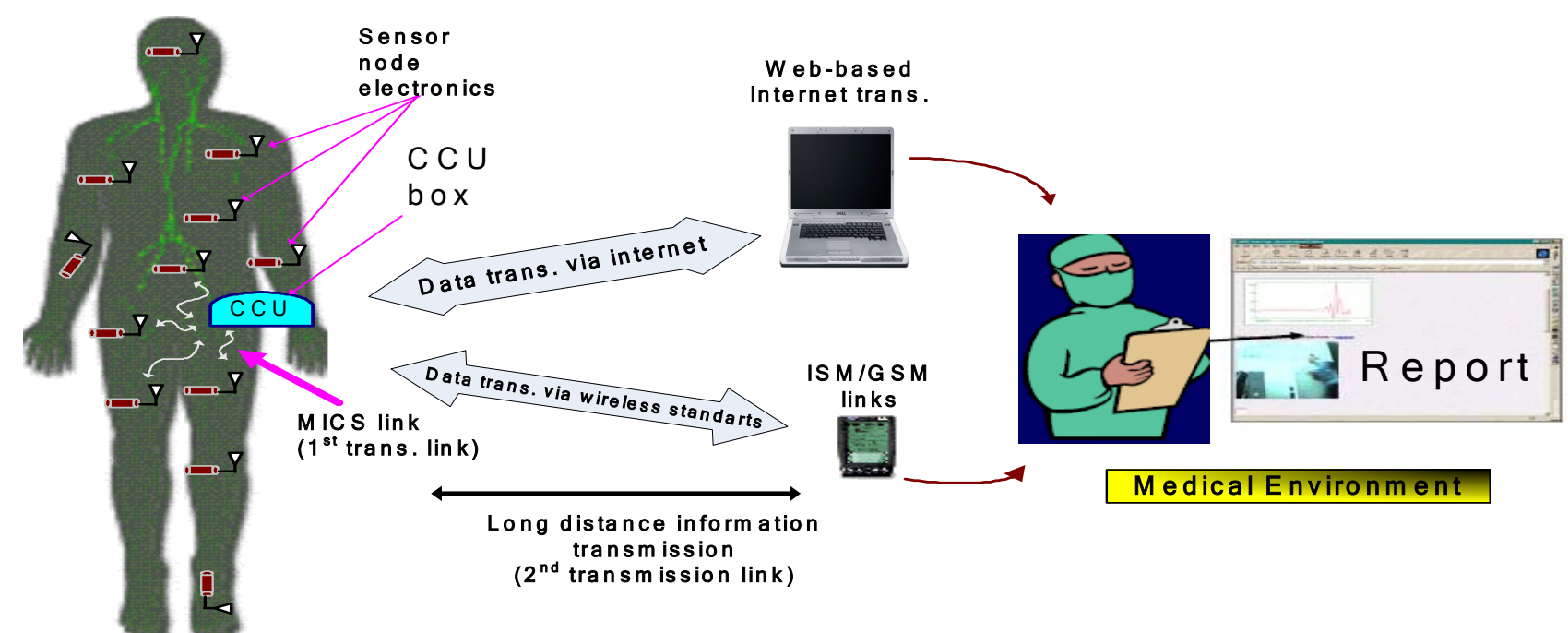

Figure-1 A wireless system detecting and transmitting signal from sensors replaced in or on the body for the proposed medical sensor network applications.

\section{SYSTEM DESCRIPTION}

The wireless Body Sensor Network comprises of sensor nodes, a Central Control Unit (CCU) and a receiver station at a medical center. The WBSN overview is as shown in Fig. 1. Sensor nodes are responsible of obtaining raw data from a human body, processing and transmitting those data via the $1^{\text {st }}$ RF Link using the MICS band to the CCU. The CCU then repackage the data and transmit to the receiver station via the $2^{\text {nd }}$ Link (an ISM band or internet). The receiver station is capable of displaying all received data on a User Display Graphic (GUI) and is also capable of storing all the data in a database of the hospital system. The CCU is targeted to be worn around a patient's waist to offer mobility to the patient. For the patients with limited mobility, the CCU will be replaced at an accessible location, at a distance of up to 10 meter in a house. In the later case, the $\mathrm{CCU}$ functions as a storage device for the information obtained from sensor nodes. It is connected to a $\mathrm{PC}$, displays real-time information and records the information to the database. The stored information can be sent via emails to the database of the hospital on a periodic basis. Pulse rate and temperature are the two vitals signs selected to be monitored in the prototype system in this paper.

Both Sensor nodes and the CCU use $3 \mathrm{~V}$ coin batteries (CR2450). Under the Live Monitoring Mode, the worst case scenario can see the battery last for at least 4.4 hours. The sensor nodes and the CCU only weights about 30 grams when fitted with batteries. The collection of data can be done one at a time from these nodes. Thus there is no time restriction and CCU initiated communication (polling) fits best for this application. Every sensor node can sleep most of the time and wake up for a very short time to see whether there is any communication session request from the $\mathrm{CCU}$ for that particular node. As a result, significant power saving is achieved and battery life is increased, which is very important for medical devices [14].

\section{SENSOR NODES DESIGN}

Sensor nodes are designed to collect raw signals from a human body. The signal from a human body is usually weak and coupled with noise. Thus, the signal should go through an amplification process to increase the signal strength. It then passes through a filtering stage to remove unwanted signals and noise. After which, it will go through an Analog to Digital Conversion (ADC) stage to be converted into digital for digital processing. The digitized signal is then processed and stored in the microprocessor (i.e. microcontroller). The microprocessor will then pack those data and transmit over the air via a transmitter (see Fig. 2).

\section{A. Pulse Rate Sensor Node Design}

The overview of the Pulse Rate Sensor Node is shown in Fig. 2. As can been seen, the pulse rate sensor node comprises of several blocks including the pulse detector, the low-noise op-amp stage, filtering, ADC, signal processing by the microcontroller and the transmitter. Microcontroller PIC16F877 and the transceiver AMIS-5210 are selected in the project because of the following reasons: overall cost saving, low-power consumption, size, and the suitability operating at MICS band and for physiological data processing. The PIC controller has 10bit and 8-bit ADC built in used to digitize the analog signal, which eliminates the need for an external off-shelf ADC.

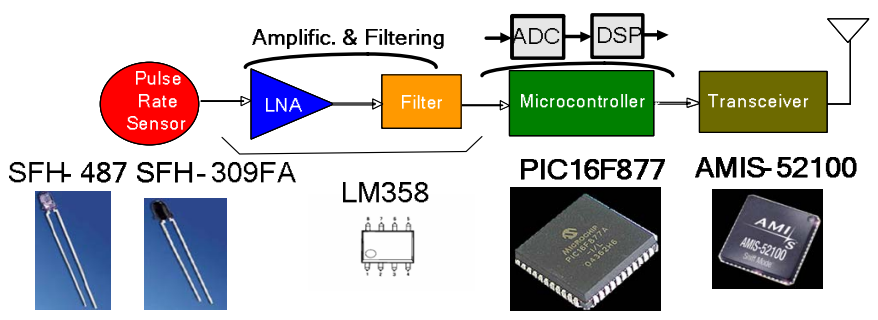

Figure 2. Block diagram of pulse rate sensor node. 


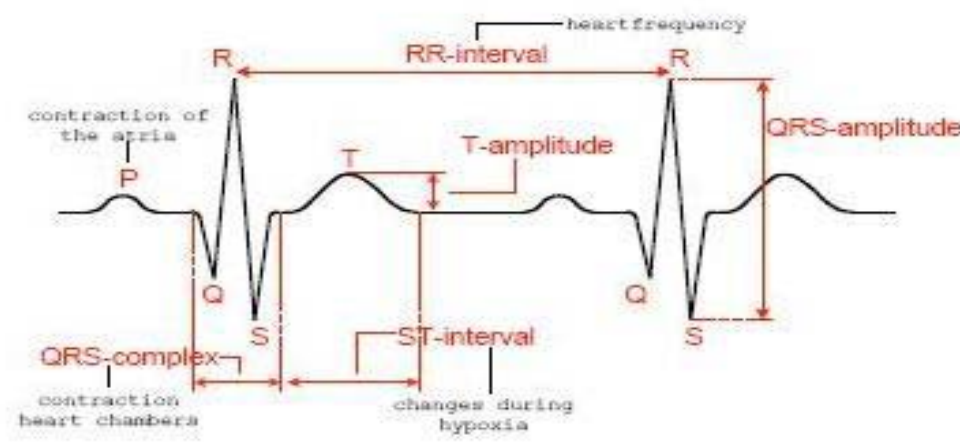

Figure 3: Complete Pulse Rate Pattern [11].

The sensor consists of an infrared emitter $(880 \mathrm{~nm})$ and a phototransistor. It is designed as described in [11]. When the light is shone through the tissues, the variation in blood volume changes the amount of light falling on the phototransistor. Pulse rate usually varies from person to person, people different age group, and may even be different for the same person at different times of the day and during different activities. When the heart beats, a pressure wave moves out along the arteries at a few meters per second. This pressure wave that can be felt at the wrist causes an increase in blood volume in the tissues which can be detected by the measuring sensor. A measuring device for such an activity is called plethysmograph. This word comes from the Greek "plethysmos" for increase and is a term used for a "fullness" (i.e. change in volume)[11].

Refer to Fig. 4 for a complete pulse rate pattern. Pulse rate or the heart frequency is calculated based on the RR-interval. Calculation of heartbeat per min requires the user to take the period between the 2 consecutive R's. Let this period be T. Then the beats per minute (bpm) is calculated as $60 / \mathrm{T}=\mathrm{bpm}$. Example, if the $1^{\text {st }} \mathrm{R}$ is at $200 \mathrm{~ms}$ and the $2^{\text {nd }} \mathrm{R}$ happens at $1000 \mathrm{~ms}$, the period is $800 \mathrm{~ms}$. Therefore the heartbeat of this person is $60 / 800 \mathrm{~ms}=75 \mathrm{bpm}$. Human heartbeat ranges between $50 \mathrm{bpm}$ to $200 \mathrm{bpm}$. The frequency range of a human heartbeat is thus between $0.83 \mathrm{~Hz}$ and $3.33 \mathrm{~Hz}$ :

$$
\begin{aligned}
50 \mathrm{bpm} & =50 / 60=0.83 \mathrm{~Hz} \\
200 \mathrm{bpm} & =200 / 60=3.33 \mathrm{~Hz}
\end{aligned}
$$

The filter stage given in Fig. 2 is designed at the lower cutoff frequency of $0.5 \mathrm{~Hz}$ and an upper cutoff frequency of $3.5 \mathrm{~Hz}$.

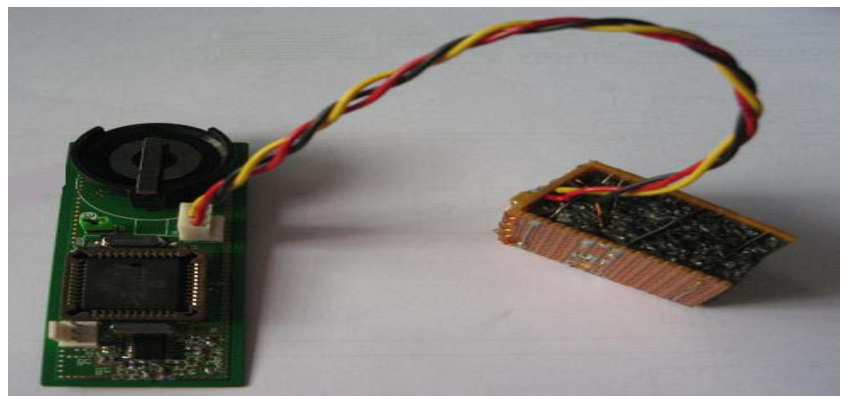

Figure 4 Hardware design of pulse rate sensor node.

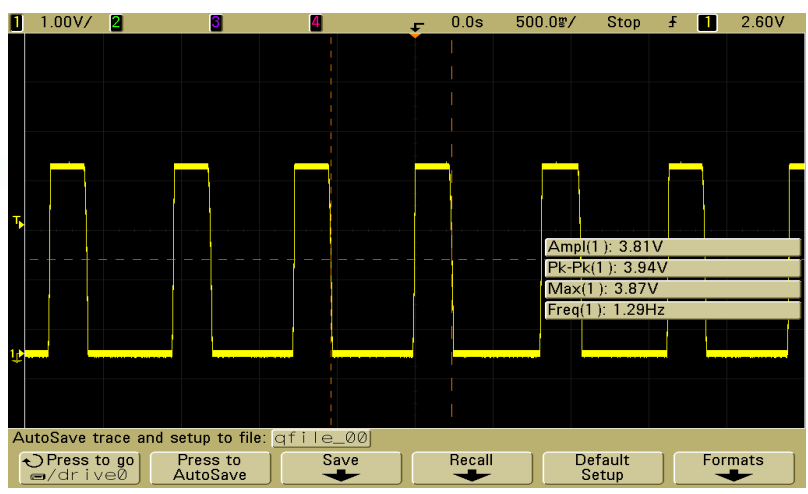

Figure 5. Detected pulse rate via the interface circuit (amplifier \& filter).

The ADC samples the input to allow calculation of pulse rate by the microcontroller. The transmitter performs data packaging and transmits data using the Manchester Coding to the CCU via the MICS band. Hardware implementation of the Pulse Rate Sensor Node is shown in Fig. 4. The Op-amp stage amplifies the input by 10000 to saturate the input signal, thus a square waveform is expected at the output of the op-amp. A sample pulse rate after the op-amp is shown in Fig. 5.

\section{B. Temperature Sensor Node Design}

The overview of the temperature sensor node is shown in Fig. 6. The temperature sensor chosen is LM35. It has a good accuracy which is $\pm 0.4{ }^{\circ} \mathrm{C}$ and draws very low-power only 60 $\mu \mathrm{W}$. Temperature sensor produces analog voltage with respect to the Celsius temperature. The ADC in the microcontroller samples the voltage and converts to a digital data for the RF transmission. The transmitter adds some overhead and package the data for transmission. Fig. 7 shows the hardware implementation of temperature sensor node.

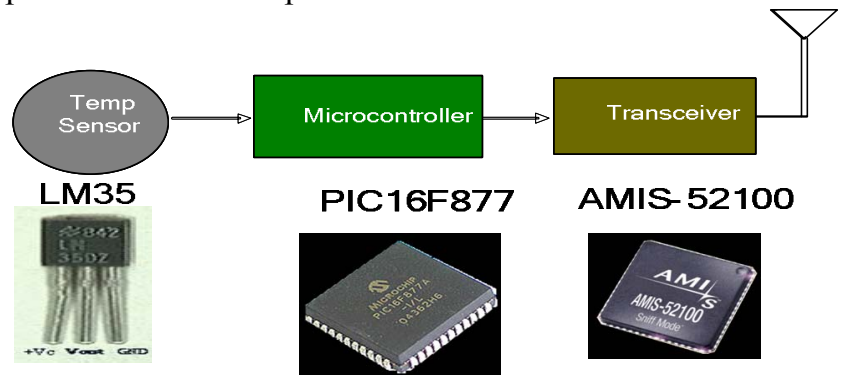

Figure 6. Overview of temperature sensor node

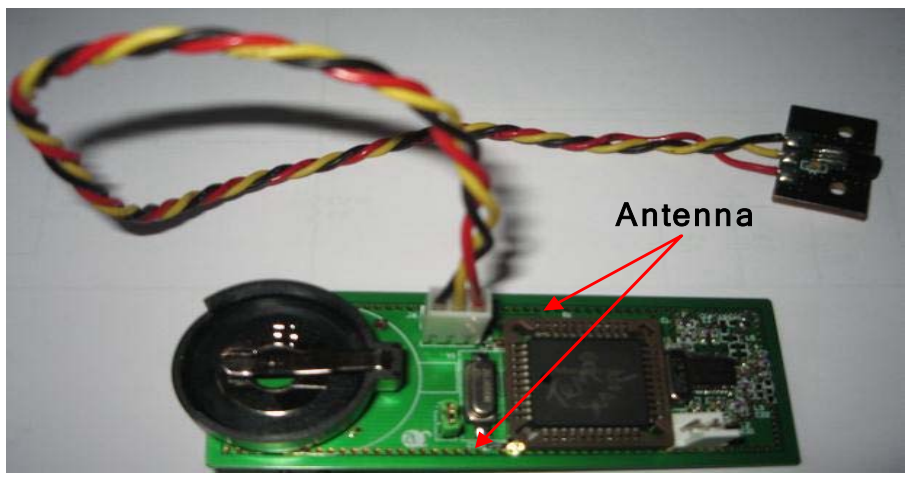

Figure 7. Hardware design of the temperature sensor node 


\section{CCU DESIGN}

The proposed block diagram of the $\mathrm{CCU}$ is shown in Fig. 8 . The primary function of the $\mathrm{CCU}$ is to collect all data from sensor nodes via the wireless MICS link and then transmit it to the Data Collection Center in the medical environment via the Internet or an ISM Band (i.e. wireless data transfer). In case of using internet facilities, the data is transferred to RS232 by the level shifter and is then sent to the PC. The target distance for the $1^{\text {st }}$ RF link (MICS Band) is 1-10 meters. The target distance for the $2^{\text {nd }}$ link (ISM Band or via the internet) is $10 \mathrm{~km}$ and more. The wireless data transfer part of the Fig. 8 is targeting to use the mobile/cellular network systems as being done by the projects in [4][5]. The incoming data is passed through the transceiver and is stored in the microprocessor. Then the data can be displayed on the screen of the PC or can be saved as a text file into a database at the same time. The $\mathrm{CCU}$ can be located at the waist of the patient or an easily accessible place. The CCU differentiates the incoming data by its address. This will help to store the correct sensor data to the correct location. When the $\mathrm{CCU}$ is connected to a computer, it is able to display real time data on a User Graphic Interface.

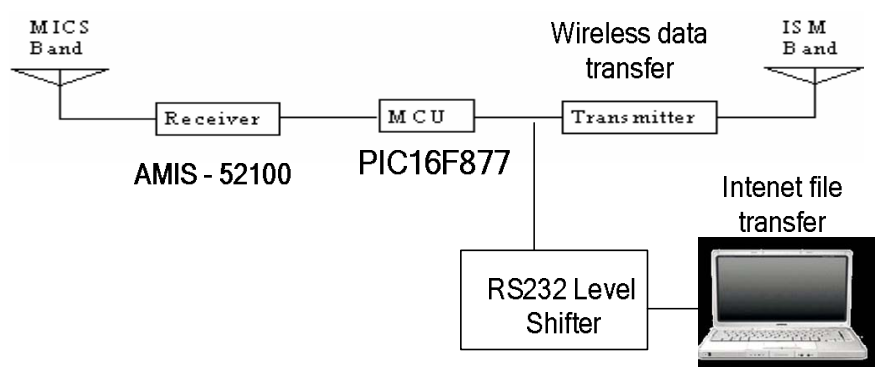

Figure 8: Overview of CCU.

\section{SOFTWARE AND FIRMWARE DESIGN}

For this body sensor network project, three major software components are implemented. One software program for a User Graphic Interface (GUI), one Firmware for the CCU, and another Firmware program at sensor nodes. The GUI software is designed to display the collected data from sensor nodes and stores the collected data as a text file. The software program also accommodates a serial communication to interact with the CCU. The GUI allows the medical personnel to enter the patient's information, diagnosis, doctor-in-charge. It displays real time graph of patient's temperature data and pulse rate. The GUI is shown in Fig. 9. The data stored in the database can be seen any time at the medical center by doctors. A sample of data storage file is shown in Fig. 10. Firmwares define the communication protocol and are required at sensor nodes, CCU, and the receiver at the data collection center. They aim to reduce the power consumption of the sensor nodes and the CCU.

In this prototype system, the temperature sensor is programmed to wake up and collect data every 10 minutes while the pulse rate sensor collects data every 5 minutes. The data will be stored in the EEPROM on the sensor nodes. For temperature sensor, sampling is performed every $100 \mathrm{~ms}$. As for the pulse rate sensor, sampling is performed every $50 \mathrm{~ms}$ for 10 seconds. The sampling of data is done through the internal $\mathrm{ADC}$ of the microcontroller. The microcontroller computes the pulse rate during these 10 seconds period and multiplies by 6 to obtain the heartbeat per minute.

In order to save power consumption, the data will not be sent every 5 or 10 minutes. Instead it will collect a block of data and send this block of data every hour. Since the transmitter drawing high current, this method will ensure the

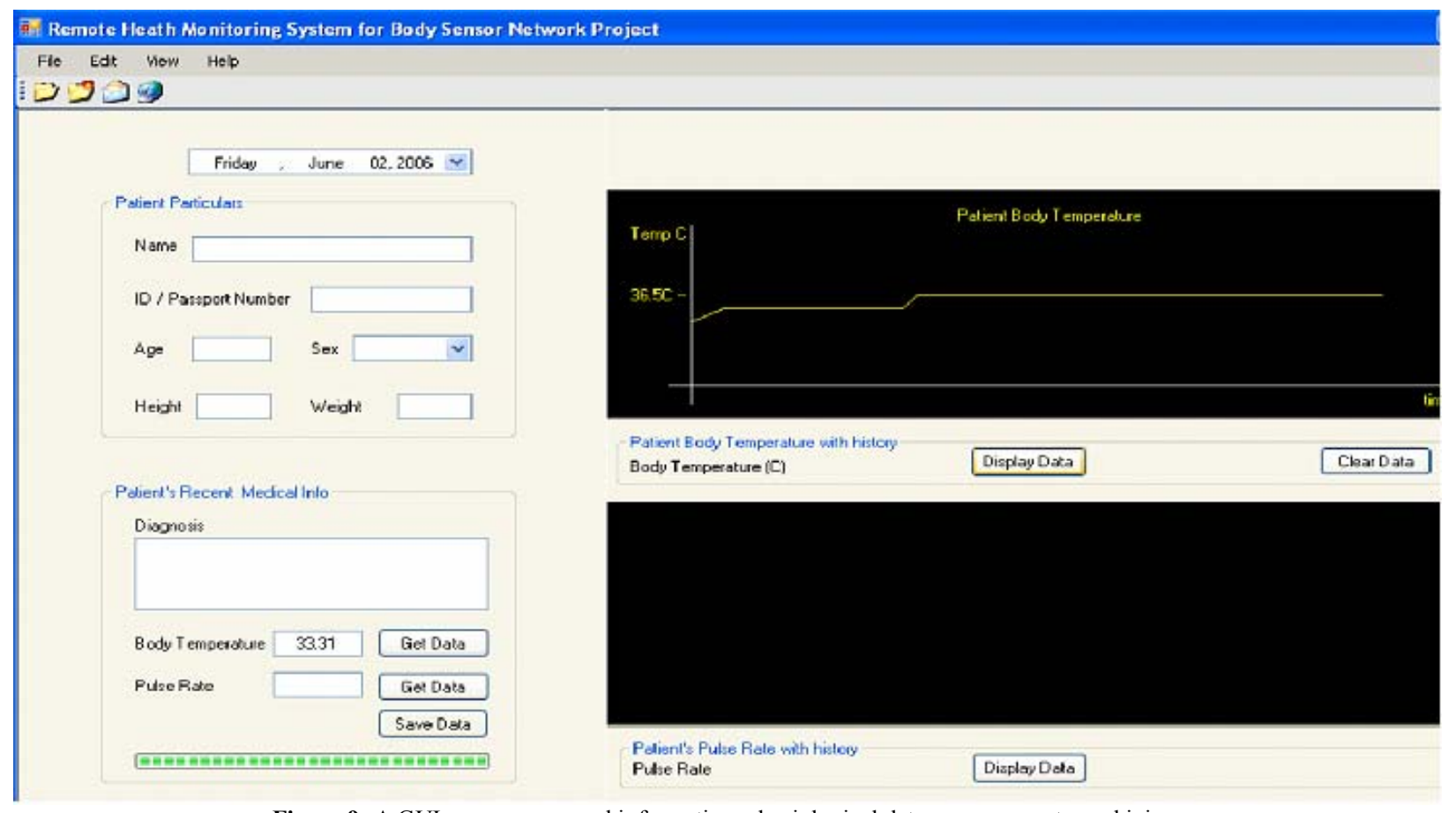

Figure 9: A GUI screen: personal information, physiological data measurements, archiving. 


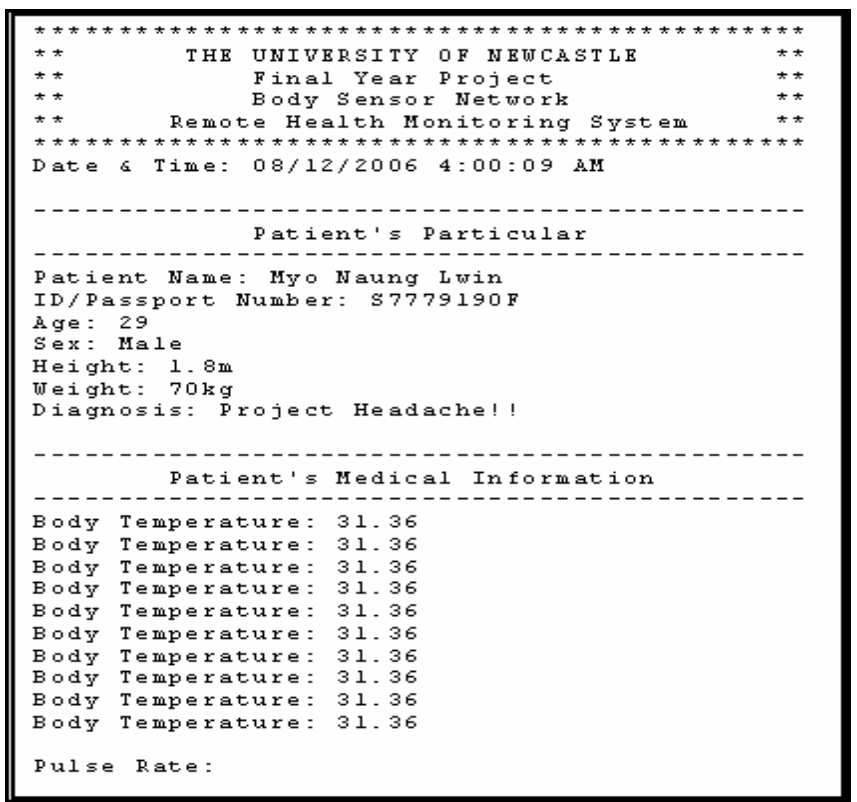

Figure 10: An example of data storage file

power consumption is minimized. The transmitter is programmed with data rate of $8 \mathrm{Kbps}$ with Manchester Coding. The transmitter power with the above setting is $25 \mathrm{~mA}$. The CCU uses low power RF Sniff mode. This mode enables the receiver to wake up at times to "sniff" received RF signals and then return to "sleep" if a signal is not detected [12]. Sniff mode current consumption is $75 \mu \mathrm{A}$ while the receive current consumption is $7.5 \mathrm{~mA}$.

The communication protocol is designed such that the central control unit becomes centralized between sensor $\mathrm{s}$ and user interface software. It includes one way wireless protocol and two-way RS 232 protocols as shown in Fig 11. The transmitter power for the MICS bad is limited by the regulation. It can be chosen maximum $25 \mu \mathrm{W}$ (i.e. EIRP=$16 \mathrm{dBm})[8][9]$. In the prototype system, transmission distance is measured for a range of transmitter power as shown in Fig. 12. The range is basically limited by the receiver sensitivity of the AMIS transceiver [12].

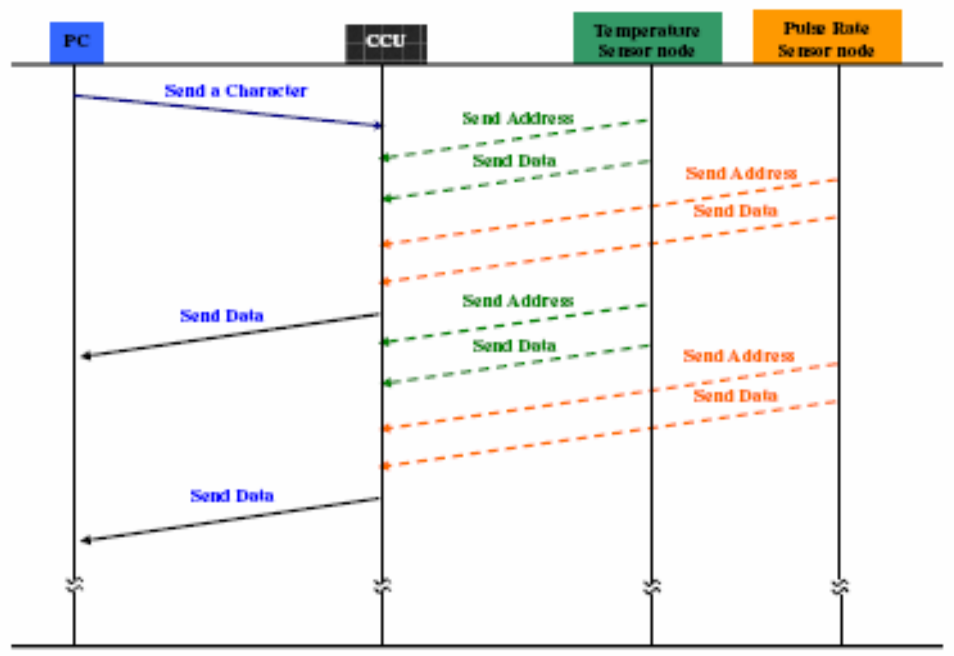

Figure 11. Communication scheme of wirless body sensor network

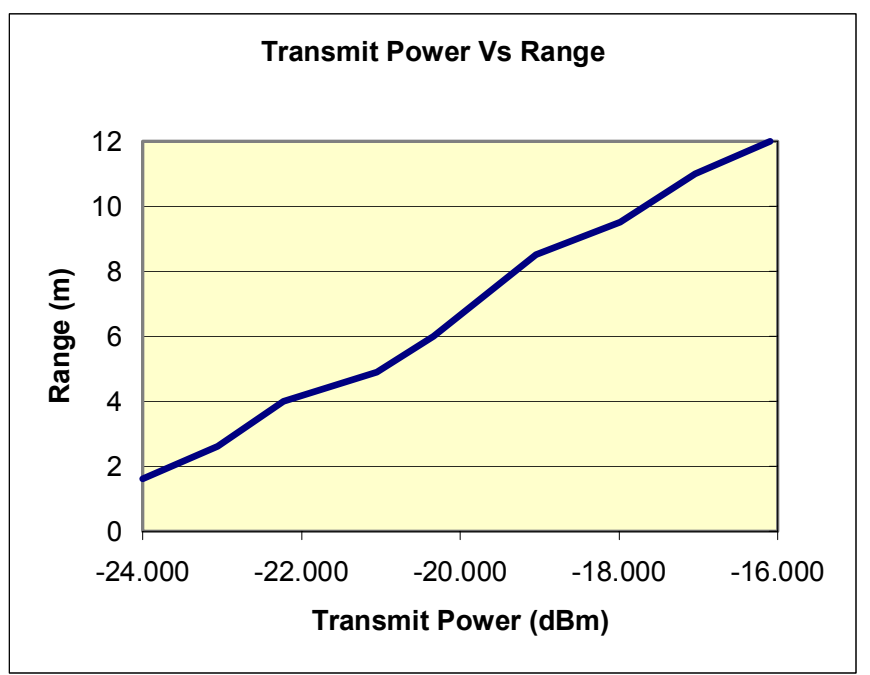

Figure 12. Measured transmission range for a arrange of different transmitter power .

The power consumption of the pulse rate sensor is the highest of the entire WBSN. Under the Live-Monitoring Mode, the current consumption of the pulse rate sensor is $126 \mathrm{~mA}$. With a battery of $560 \mathrm{mAh}$, the Pulse Rate Sensor can last 4.4hours continuously. We investigated the off-time vs battery lifespan and found that a longer off-time will result in a longer battery lifespan. The results for the maximum allowable transmitted power are shown in Fig. 13. The temperature sensor battery can last up to 12.9 hours while the battery of the CCU can last up to 14 .6hours. Since the receiver station is not mobile, it is designed to draw power from a communication port of a computer. The power consumption is further reduced by keeping sensor nodes in sleep mode for longer durations (i.e. increasing off-time).

\section{CONCLUSION AND FUTURE WORK}

A low cost, power efficient wireless body sensor network system (WBSN) has been successfully implemented for patient monitoring. The prototype system includes two sensor nodes and a central control unit. The sensor nodes are able to transmit data to the CCU upto $12 \mathrm{~m}$ range with a $-17.3 \mathrm{dBm}$ EIRP. The MICS band requires a $-16 \mathrm{dBm}$ EIRP for transmit power [8]. The CCU is able to display data on the GUI. The sensors designed are calibrated to obtain data accuracy. The temperature sensor is calibrated with a Mini-Logger (GL500) while the pulse rate sensor is calibrated with a commercial pulse rate sensor approved by health authorities. The manufacturing cost of the entire system is USD200 with each sensor node costing around USD 60. The CCU costs USD50 while the receiver station costs USD30.

The work in this paper has focused on an external sensor node design for medical monitoring. The goal of future work is additionally to design a miniaturized sensor node that is small enough to make them implantable. The CCU will initiate 


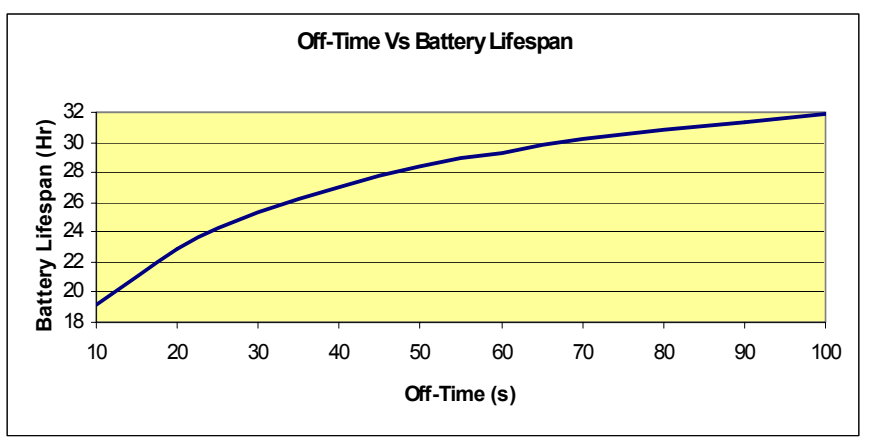

Temperature Sensor Power Consumption

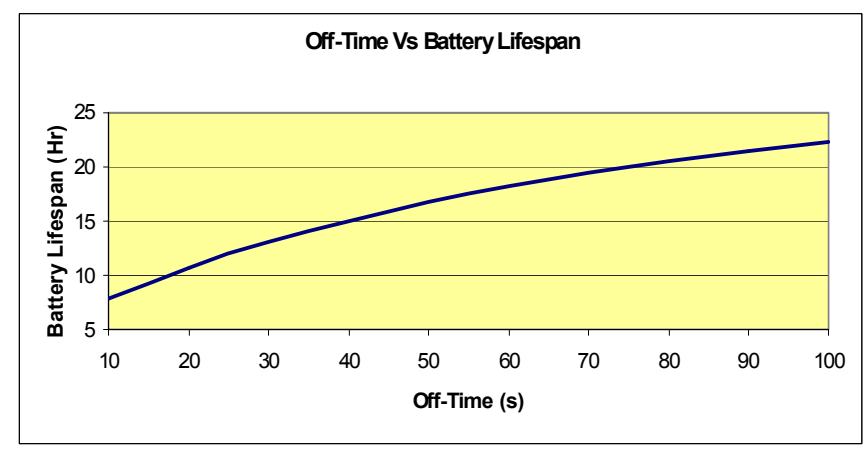

Pulse Rate Sensor Power Consumption

Figure 13. Battery lifetime measurement of sensor nodes

the sensor node to perform a measurement only when it is necessary. In this communication we can keep the power consumption significantly low so that the battery life can last several years [14]. The microprocessor (microcontroller) used here is too powerful, in future a lower range microprocessor will be used for both the implanted or external sensor nodes to reduce the size as well as the power consumption. Currently a custom chips are being developed [14][15] especially targeting towards fully implantable sensor nodes. Future work also involves designing more sensors to monitor other vital signs such as blood pressure, oxygen saturation level, ECG, etc.

\section{REFERENCES}

[1] Emily Yoffe, "Doctors Are Reminded, 'Wash Up!'," NEW YORK TIMES November 9, 1999, pg. F-1.

[2] C. C. Y. Poon, Y-T Zhang, S-D Bao, "A novel biometrics method to secure wireless body area sensor networks for telemedicine and mhealth," IEEE Communications Magazine, vol. 44, pp. 73-81, April 2006 .

[3] R. Fischer et al. SMART: Scalable Medical Alert and Response Technology. http://smart.csail.mit.edu/.

[4] T. Gao et al. "Vital Signs Monitoring and Patient Tracking Over a Wireless Network," IEEE-EMBS 27th Annual Int. Conference of the Eng. in Medicine and Biology, Sept. 2005, Page(s):102 - 105.

[5] U. Anliker, " AMON: a werable multiparameter medical monitoring and alert system, in IEEE Trans. Information Tech. In Biomedicine, vol. 8., Dec 2004

[6] F. Magrabi, N. H. Lovell, and B. G. Celler, "Web based longitudinal ECG monitoring," Proc. 20th Annu. Int. Conf. IEEE EMBS, vol. 20, no. 3, pp. 1155-1158, 1998.

[7] S. Park, et al., "Real-time monitoring of patient on remote sites," Proc. 20th Annu. Int. Conf. IEEE EMBS, vol. 20, no. 3, pp. 1321-1325, 1998.

[8] FCC Rules and Regulations, "MICS Band Plan," Table of Frequency Allocations, Part 95, Jan. 2003.

[9] Australian Communications Authority, Radio Frequency Planning Group, "Planning for Medical Implant Communications Systems \& Related Devices", Proposals Paper, Oct. 2003.

[10] W. G. Scanlon, J. B. Burns, and N. E. Evans, "Radiowave propagation from a tissue implanted source at $418 \mathrm{MHz}$ and 916. $5 \mathrm{MHz}$," IEEE Trans. Biomedical Engineering, vol.47, pp.527-534, April 2000.

[11] Dr G J Compton, Calculating the heart rate with a pulse plethysmograph, School of Science, Nottingham Trent University,UK.

http://www.picotech.com/experiments/calculating_heart_rate/
[12] AMIS 52100 Low Power Transceiver with Clock and Data Recovery Data Sheet.

[13] http://www.wirelessis.com

[14] A. Tekin, M. R. Yuce, and W. Liu, "A Low power MICS band transceiver architecture for implantable devices," in Proc. IEEE Wireless and Microwave Tecnology, pp. 55-58, April 2005.

[15] A. Tekin, M. R. Yuce and W. Liu, " Integrated VCO Design for MICS Transceivers," in Proc. IEEE Custom Integrated Circuits Conference (CICC'06), pp. 765-768, Sep., 2006. 